\title{
Effect of a 12 week low carbohydrate ketogenic diet versus a high carbohydrate diet on blood count indicators of iron status in male endurance athletes
}

\author{
F. McSwiney ${ }^{1}$, B. Wardrop ${ }^{1}$, J. Volek $^{2}$ and L. Doyle ${ }^{1}$ \\ ${ }^{1}$ Department of Health, Sport and Exercise Science, Waterford Institute of Technology, Cork Road, Waterford and \\ ${ }^{2}$ Kinesiology Program, Department of Human Sciences, The Ohio State University, Columbus, Ohio, USA
}

Low carbohydrate ketogenic diets (LCKD) where carbohydrate intake is $<50 \mathrm{~g} / \mathrm{d}$ have become popular is many populations, including endurance athletes ${ }^{(1)}$. The complete effect of this diet on nutrient absorption and its potential to influence health has not been fully uncovered $^{(2)}$. A 14 day ketogenic diet in females demonstrated no effect on RBC, Hb or Hct levels in healthy females ${ }^{(3)}$, but longer duration research in an athletic population is lacking. The aim of the current research is to examine the effect of a LCKD versus a high carbohydrate diet, on blood count indicators of iron status in endurance athletes.

Following ethical approval 19 participants selected into a high carbohydrate (HC) $(65 \% \mathrm{kcal} \mathrm{CHO,} 20 \% \mathrm{kcal}$ fat, $14 \% \mathrm{kcal}$ protein) or LCKD (>75\% kcal fat, 10-15\% kcal protein and $<50 \mathrm{~g} / \mathrm{d}$ CHO) group for 12 weeks. Participants also completed an endurance training protocol during the trial. Whole blood samples were analysed using the haematology analyser before and after selection into each dietary group. RM ANOVA was used to assess changes in indicators of iron status in participants over time. Paired t-tests assessed changed within group over time.

\begin{tabular}{|c|c|c|c|c|c|c|c|c|c|c|c|}
\hline & \multicolumn{4}{|c|}{$\mathrm{HC} \operatorname{diet}(n=10)$} & \multicolumn{4}{|c|}{$\underline{\text { LCKD diet }(n=9)}$} & \multirow{2}{*}{\multicolumn{3}{|c|}{$\frac{\mathrm{RM} \text { ANOVA }}{P \text { Value }}$}} \\
\hline & \multicolumn{2}{|c|}{ Pre } & \multicolumn{2}{|l|}{ Post } & \multicolumn{2}{|c|}{ Pre } & \multicolumn{2}{|l|}{ Post } & & & \\
\hline & Mean & SD & Mean & SD & Mean & SD & Mean & SD & Time & Group & Group \\
\hline $\mathrm{RBC}\left(10^{-6} \mu \mathrm{l}\right)$ & $4 \cdot 59$ & $0 \cdot 33$ & $4 \cdot 30$ & 0.63 & 4.67 & $0 \cdot 42$ & $4 \cdot 44$ & 0.61 & $0.038^{*}$ & 0.620 & 0.783 \\
\hline $\mathrm{Hb}(\mathrm{g} / \mathrm{dl})$ & $14 \cdot 15$ & $1 \cdot 25$ & $13 \cdot 36$ & $1 \cdot 87$ & $14 \cdot 29$ & 0.99 & $12 \cdot 95$ & $1.62^{\mathrm{a}}$ & $0.013^{*}$ & $0 \cdot 816$ & $0 \cdot 487$ \\
\hline Het $(\%)$ & 0.41 & 0.03 & $0 \cdot 38$ & $0 \cdot 05^{\mathrm{a}}$ & 0.41 & $0 \cdot 03$ & $0 \cdot 39$ & $0 \cdot 05$ & $0.015^{*}$ & $0 \cdot 846$ & $0 \cdot 744$ \\
\hline MCV (fL) & $90 \cdot 47$ & $4 \cdot 52$ & $89 \cdot 35$ & $4 \cdot 78$ & $89 \cdot 19$ & $3 \cdot 14$ & $88 \cdot 31$ & $2 \cdot 40$ & 0.082 & $0 \cdot 430$ & $0 \cdot 825$ \\
\hline MCH (pg/cell) & $30 \cdot 81$ & 1.78 & $31 \cdot 14$ & $2 \cdot 00$ & $30 \cdot 88$ & 1.75 & $29 \cdot 27$ & $1.63^{\mathrm{a}}$ & $0 \cdot 113$ & $0 \cdot 239$ & $0.021 *$ \\
\hline $\mathrm{MCHC}(\mathrm{g} / \mathrm{dl})$ & $33 \cdot 66$ & 1.95 & $34 \cdot 85$ & 1.52 & $34 \cdot 59$ & 0.87 & $33 \cdot 14$ & $1 \cdot 33^{\mathrm{a}}$ & 0.801 & 0.417 & $0.017^{*}$ \\
\hline RDW (\%) & $12 \cdot 65$ & $0 \cdot 70$ & $12 \cdot 52$ & $0 \cdot 69$ & $12 \cdot 89$ & 0.44 & $13 \cdot 83$ & $2 \cdot 34$ & 0.253 & $0 \cdot 116$ & $0 \cdot 137$ \\
\hline
\end{tabular}

$\mathrm{RBC}$ - red blood cell; $\mathrm{Hb}$ - haemoglobin; Hct - haematocrit; MCV - mean corpuscular volume; MCH - mean corpuscular haemoglobin; MCHC - mean corpuscular

haemoglobin concentration; red cell distribution width.

* Significant difference at $P<0.05 ;{ }^{a}$ Significant difference within group over time at $P<0.05$.

$\mathrm{RBC}, \mathrm{Hb}$, and Hct significantly decreased over time. Within group Hct decreased between weeks 1 and 12 in the HC participants and $\mathrm{Hb}, \mathrm{MCH}$ and $\mathrm{MCHC}$ decreased within the LCKD participants.

Endurance training has been associated with increased plasma volume with exercise induced inflammation also implicated as a possible cause for iron deficiency in athletes ${ }^{(4)}$. It is possible the endurance training added during this trial had a similar effect on iron status within these participants. However it does not explain the difference in $\mathrm{Hb}, \mathrm{MCH}$ and MCHC between HC and LCKD participants. Due to the effect iron status has on oxygen transport to muscle, further research is warranted to investigate the effect of a ketogenic diet on iron status in endurance athletes.

1. Volek JS, Noakes T \& Phinney SD (2015) Eur J Sp Sci 15, 13-20.

2. Paoli A, Rubini A, Volek JS et al. (2013) Eur J Clin Nutr 67, 789-796.

3. Nazarewicz RR, Ziolkowski W, Vaccaro PS et al. (2007) Rejuvenation Res 10, 435-439.

4. Hinton PS (2014) Appl Physiol Nutr Metab 39, 1012-1018. 